\title{
Myocardial protective effect by ulinastatin via an anti- inflammatory response after regional ischemia/ reperfusion injury in an in vivo rat heart model
}

\author{
Il-Woo Shin ${ }^{1,3}$, In-Seok Jang ${ }^{2,3}$, Seung-Min Lee ${ }^{1}$, Kyeong-Eon Park ${ }^{1}$, Seong-Ho Ok ${ }^{1}$, Ju-Tae Sohn ${ }^{1,3}$, \\ Heon-Keun Lee ${ }^{1,3}$, and Young-Kyun Chung ${ }^{1,3}$ \\ Departments of ${ }^{1}$ Anesthesiology and Pain Medicine, ${ }^{2}$ Cardiothoracic and Vascular Surgery, ${ }^{3}$ Institute of Health Sciences, Gyeongsang \\ National University College of Medicine, Jinju, Korea
}

Background: Ulinastatin has anti-inflammatory properties and protects organs from ischemia/reperfusioninduced injury. The aim of this study was to investigate whether ulinastatin provides a protective effect on a regional myocardial ischemia/reperfusion injury in an in vivo rat heart model and to determine whether the antiinflammatory response is related to its myocardial protective effect.

Methods: Rats were randomized to two groups. One group is received ulinastatin (50,000 U/ kg or 100,000 U/ kg) diluted in normal saline and the other group is received normal saline, which was administered intraperitoneally $30 \mathrm{~min}$ before the ischemic insult. Reperfusion after $30 \mathrm{~min}$ of ischemia of the left coronary artery territory was applied. Hemodynamic measurements were recorded serially during $6 \mathrm{~h}$ after reperfusion. After the $6 \mathrm{~h}$ reperfusion, myocardial infarct size, cardiac enzymes, myeloperoxidase activity, and inflammatory cytokine levels were compared between the ulinastatin treated and untreated groups.

Results: Ulinastatin improved cardiac function and reduced infarct size after regional ischemia/reperfusion injury. Ulinastatin significantly attenuated tumor necrosis factor- $\alpha$ expression and reduced myeloperoxidase activity.

Conclusions: Ulinastatin showed a myocardial protective effect after regional ischemia/reperfusion injury in an in vivo rat heart model. This protective effect of ulinastatin might be related in part to ulinastatin's ability to inhibit myeloperoxidase activity and decrease expression of tumor necrosis factor- $\alpha$. (Korean J Anesthesiol 2011; 61: 499-505)

Key Words: Inflammation, Ischemia/reperfusion, Myocardium, Ulinastatin.

Received: October 13, 2010. Revised: 1st, December 7, 2010; 2nd, April 1, 2011. Accepted: May 19, 2011.

Corresponding author: Young-Kyun Chung, M.D., Ph.D., Department of Anesthesiology and Pain Medicine, Gyeongsang National University College of Medicine, 92, Chilam-dong, Jinju 660-751, Korea. Tel: 82-55-750-8141, Fax: 82-55-750-8142, E-mail: ykchung@nongae.gsnu.ac.kr (c) This is an open-access article distributed under the terms of the Creative Commons Attribution Non-Commercial License (http:// creativecommons.org/licenses/by-nc/3.0/), which permits unrestricted non-commercial use, distribution, and reproduction in any medium, provided the original work is properly cited. 


\section{Introduction}

Since protease activation is one of known mechanisms for developing ischemic injury, inhibiting this protease activation has been proposed to attenuate post-ischemic injury [1-4]. Proteases, which are secreted from activated neutrophils during acute inflammatory phase, degrade the extracellular matrix and lead to tissue injury. While several protease inhibitors showed a myocardial protective effect in ischemia/reperfusion (I/R) injury models [5-7], ulinastatin, a protease inhibitor derived from human urine, also showed organ protective effect against I/R injury in the brain [8,9], liver [10,11], kidney [12], lung [13], and heart $[6,7]$. Previous investigations have showed that ulinastatin reduces the release of elastase from neutrophils and inhibits the accumulation of neutrophils in various I/R injury models $[5,8,10,13]$ meaning that ulinastatin's protective effect is due to its anti-inflammatory response.

In this study, the effect of ulinastatin on several physiological and biochemical variables in an in vivo rat heart model of regional I/R injury was performed to determine whether ulinastatin's myocardial protective effects were due to an antiinflammatory response.

\section{Materials and Methods}

\section{Animal preparation}

Male Sprague-Dawley rats (Sam Tako Inc., Osan, South Korea) weighing between 220 and $250 \mathrm{~g}$ were used for this study. All animals were maintained in accordance with the Guidelines for the Care and Use of Laboratory Animals published by the US National Institutes of Health in 1996. The protocol was approved by the Animal Research Committee.

Animal preparation and surgery were performed as previously described [14]. Animals received general anesthesia with an intramuscular injection of $15 \mathrm{mg} / \mathrm{kg}$ of tiletamine/zolazepam (Zoletil50 ${ }^{\circledR}$, Virbac Lab., Carros, France) and $8 \mathrm{mg} / \mathrm{kg}$ of xylazine (Rompun $^{\circledR}$, Bayer, Seoul, South Korea). Additionally, $15 \mathrm{mg} / \mathrm{kg}$ of tiletamine/zolazepam and $8 \mathrm{mg} / \mathrm{kg}$ of xylazine was injected to maintain the level of anesthesia after $3 \mathrm{~h}$ reperfusion. If the tail moved (i.e. a sign of awakening), an additional $7.5 \mathrm{mg} / \mathrm{kg}$ of tiletamine/zolazepam and $4 \mathrm{mg} / \mathrm{kg}$ of xylazine was injected to maintain the level of anesthesia. The trachea was intubated, and then lungs were mechanically ventilated (tidal volume, 8 $\mathrm{ml} / \mathrm{kg}$; respiratory rate, 80 strokes/min) with ambient air using a volume-controlled rodent ventilator (Harvard Rodent Ventilator Model 607, Harvard, Holliston, MA, USA). The arterial blood pH and gases were maintained within normal physiological limits (pH, 7.35-7.45; $\mathrm{PaCO}_{2}$, 30-40 mmHg; $\mathrm{PaO}_{2}, 60-100$ mmHg).

A paramedian sternotomy was performed, and the pericar- dium was opened to expose the heart. For the I/R experiments, a 4-0 black silk was passed around the left coronary artery (LCA) territory to induce regional myocardial ischemia. The coronary artery was occluded by pulling a snare through a small vinyl chloride tube and clamping it with a mosquito haemostat. Reperfusion was achieved by releasing the clamp. Myocardial ischemia was confirmed by the appearance of regional epicardial cyanosis over the myocardial surface and by arrhythmia. After $30 \mathrm{~min}$ of ischemia, the myocardium was reperfused by loosening the snare, which was maintained for $6 \mathrm{~h}$ of reperfusion. Successful reperfusion was confirmed by the disappearance of epicardial cyanosis and the production of epicardial hyperaemia and arrhythmia after releasing the snare. Body temperature was monitored with a rectal thermometer (Sirecust 1260, Siemens Medical Electronics, MA, USA) and maintained at $36-38^{\circ} \mathrm{C}$ with an electrical heating pad.

\section{Experimental protocols}

The animals were divided randomly into two sets of experiments. In the first set of experiments, animals were divided into three groups as follows: (1) I/R + Normal Saline (NS) group rats in which $1 \mathrm{ml} \mathrm{NS}$ was administered intraperitoneally $30 \mathrm{~min}$ before the ischemic insult $(\mathrm{n}=10),(2) \mathrm{I} / \mathrm{R}+\mathrm{U} 50$ group - rats in which 50,000 U/kg ulinastatin (Ulistin ${ }^{\circledR}$, Hanlim, Seoul, South Korea) diluted with NS was administered intraperitoneally 30 min before the ischemic insult $(n=10)$, and $(3) I / R+U 100$ group - rats in which 100,000 U/kg ulinastatin diluted with NS was administered intraperitoneally $30 \mathrm{~min}$ before the ischemic insult $(\mathrm{n}=10)$. Rats underwent a $30 \mathrm{~min}$ ischemia followed by reperfusion of the LCA territory. Hemodynamic measurements were recorded serially ( $30 \mathrm{~min}, 1 \mathrm{~h}, 1 \mathrm{~h} 30 \mathrm{~min}, 2 \mathrm{~h}, 6 \mathrm{~h}$ ) during the $6 \mathrm{~h}$ of reperfusion, and myocardial infarct size was measured.

In the second set of experiments, animals were divided into five groups as follows: (1) sham + NS group - rats in which 1 $\mathrm{ml}$ NS was administered intraperitoneally $30 \mathrm{~min}$ before the sham operation $(n=6)$, (2) sham + U100 group - rats in which $100,000 \mathrm{U} / \mathrm{kg}$ ulinastatin diluted with NS was administered intraperitoneally $30 \mathrm{~min}$ before the sham operation $(\mathrm{n}=6)$, (3) I/R + NS group - rats in which $1 \mathrm{ml}$ NS was administered intraperitoneally $30 \mathrm{~min}$ before the ischemic insult $(\mathrm{n}=6),(4)$ $\mathrm{I} / \mathrm{R}+\mathrm{U} 50$ group - rats in which 50,000 $\mathrm{U} / \mathrm{kg}$ ulinastatin diluted with NS was administered intraperitoneally $30 \mathrm{~min}$ before the ischemic insult $(\mathrm{n}=6)$, and (5) I/R + U100 group - rats in which $100,000 \mathrm{U} / \mathrm{kg}$ ulinastatin diluted by NS was administered intraperitoneally $30 \mathrm{~min}$ before the ischemic insult $(\mathrm{n}=6)$. All animals were exposed to $30 \mathrm{~min}$ ischemia followed by $6 \mathrm{~h}$ of reperfusion. The sham group had paramedian sternotomy and pericardiotomy performed without the LCA occlusion. The 
thoracic cavity was opened to expose the heart for sampling of 3 $\mathrm{ml}$ blood from the right ventricle. Blood samples were collected in heparin containing tubes or conical tubes to measure cardiac troponin-I (cTnI), creatine kinase (CK) concentration, tumor necrosis factor (TNF)- $\alpha$, and myeloperoxidase (MPO) activity. The entire sample was centrifuged, and serum and/or plasma were collected and frozen at $-70^{\circ} \mathrm{C}$ until analysed.

\section{Measurement of hemodynamic variables and myocardial contractility}

The right common carotid artery was cannulated with a 2-Fr Millar catheter (Model SPR-407, Millar Instruments Inc., Houston, TX, USA) and advanced into the left ventricle (LV) to measure baseline hemodynamic function (heart rate [HR], left ventricular end diastolic pressure [LVEDP], the maximum rate of intraventricular pressure increase $\left[+\mathrm{dP} / \mathrm{dt}_{\max }\right]$, and the maximum rate of intraventricular pressure decrease $[-\mathrm{dP} /$ $\left.\mathrm{dt}_{\max }\right]$ ). Hemodynamic measurements were recorded serially (30 $\min , 1 \mathrm{~h}, 1 \mathrm{~h} 30 \mathrm{~min}, 2 \mathrm{~h}, 6 \mathrm{~h}$ ) during the $6 \mathrm{~h}$ of reperfusion.

\section{Area at risk and infarct size determination}

After cardiac function analysis, the LCA was occluded again and $2 \mathrm{ml}$ of $1 \%$ Evans blue dye was injected into the pulmonary artery to facilitate area at risk (AAR) determination as described in a previous report [14]. The perfused myocardium was stained with Evans blue dye, whereas the occluded vascular bed was not. The atrium, right ventricle, and major vessels were removed from the heart, and the AAR was separated from the nonischemic area. The AAR was cut into small pieces and incubated in $2 \%$ 2,3,5-triphenyltetrazolium chloride (TTC) at $37^{\circ} \mathrm{C}$ for 20 min. The non-infarcted myocardium stained a deep red color with TTC, and the infarcted area (IA) remained pale yellow. The sections were fixed for $24 \mathrm{~h}$ in $2 \%$ formalin, and the $\mathrm{LV}$, AAR, and IA weights were determined. The AAR and IA were expressed as a percentage of the LV and AAR weights, respectively.

\section{cTnl and CK measurements}

Serum levels of cTnI were determined by an enzyme linked immunosorbent assay (ELISA) kit from Life Diagnostics (cat. no. 2010-2-HS, Life Diagnostics Inc., Wester Chester, PA, USA) as described previously [14]. The cTnI activity was detected by spectrophotometry (Infinite ${ }^{\circledR}$ F200, Tecan Group Ltd., Männedorf, Switzerland) at $460 \mathrm{~nm}$. Serum levels of CK were determined by EnzyChrom ${ }^{\mathrm{TM}}$ creatine kinase assay kit (ECPK100, BioAssay Systems, CA, USA). CK activities were detected by colorimetric determination at $340 \mathrm{~nm}$.

\section{Measurement of MPO and TNF- $\alpha$ activities}

Plasma level of MPO were determined by rat MPO ELISA test kit (Hbt HK 105, Hycult biotechnology b.v., Uden, Netherlands) according to the manufacturer as described previously [15]. MPO activities were detected to determine neutrophil accumulation by spectrophotometry (Infinite ${ }^{\circledR}$ F200, Tecan Group Ltd., Männedorf, Switzerland) at $460 \mathrm{~nm}$ after reaction of the plasma sample at MPO antibody-coated plate. Plasma concentrations of immunoreactive TNF- $\alpha$ were determined with an ELISA kit (RAT00, R\&D Systems inc., Minneapolis, MN, USA) according to the manufacturer's protocol as described previously [15]. This assay employs the quantitative sandwich enzyme immunoassay technique. Plasma was reacted with the assay reagents in the TNF- $\alpha$ kit and analyzed by spectrophotometry (Infinite ${ }^{\circledR}$ F200, Tecan Group Ltd., Männedorf, Switzerland) at an absorbance of $460 \mathrm{~nm}$.

\section{Statistical analysis}

All values are expressed as the means \pm SD. All statistical analyses were performed using SPSS software (ver. 12.0 for Windows, SPSS Inc., Chicago, IL, USA). The differences between groups were evaluated using a Friedman one-way analysis of variance and a Tukey's multiple comparison post hoc test. Statistical significance was defined as $\mathrm{P}<0.05$.

\section{Results}

\section{Hemodynamic function following I/R injury}

The hemodynamic measurements recorded serially during the $6 \mathrm{~h}$ after reperfusion revealed substantial LV dysfunction as demonstrated by decreased $\pm \mathrm{dP} / \mathrm{dt}_{\max }$ after I/R injury in the I/R + NS group compared to the basal hemodynamic function (Fig. 1). The LVEDP in the I/R + U100 group $(12 \pm 2 \mathrm{mmHg}, \mathrm{P}=0.012)$ showed significant difference compared to the $\mathrm{I} / \mathrm{R}+\mathrm{NS}$ group $(15 \pm 2$ $\mathrm{mmHg}$ ) after $6 \mathrm{~h}$ of reperfusion. The $\pm \mathrm{dP} / \mathrm{dt}_{\max }$ in the $\mathrm{I} / \mathrm{R}+\mathrm{U} 100$ group $\left(+\mathrm{dP} / \mathrm{dt}_{\max }:+3,088 \pm 362 \mathrm{mmHg} ; \mathrm{P}<0.001,-\mathrm{dP} / \mathrm{dt}_{\max }\right.$ : $-2,157 \pm 290 \mathrm{mmHg} ; \mathrm{P}=0.011)$ showed significant difference compared to the I/R $+\mathrm{NS}$ group $\left(+\mathrm{dP} / \mathrm{dt}_{\text {max }}:+3,972 \pm 227 \mathrm{mmHg} /\right.$ $\mathrm{sec},-\mathrm{dP} / \mathrm{dt}_{\text {max }}:-3,412 \pm 248 \mathrm{mmHg}$ ) after $6 \mathrm{~h}$ of reperfusion. The HR was not significant difference between the groups.

\section{Myocardial infarct size}

The AAR/LV induced by occluding the LCA territory was not significantlt different between the three groups. The IA/ AAR in the I/R + NS group $(49 \pm 7 \%)$ was significantly different compared to the I/R + U50 $(40 \pm 7 \%, \mathrm{P}=0.016)$ and I/R + U100 

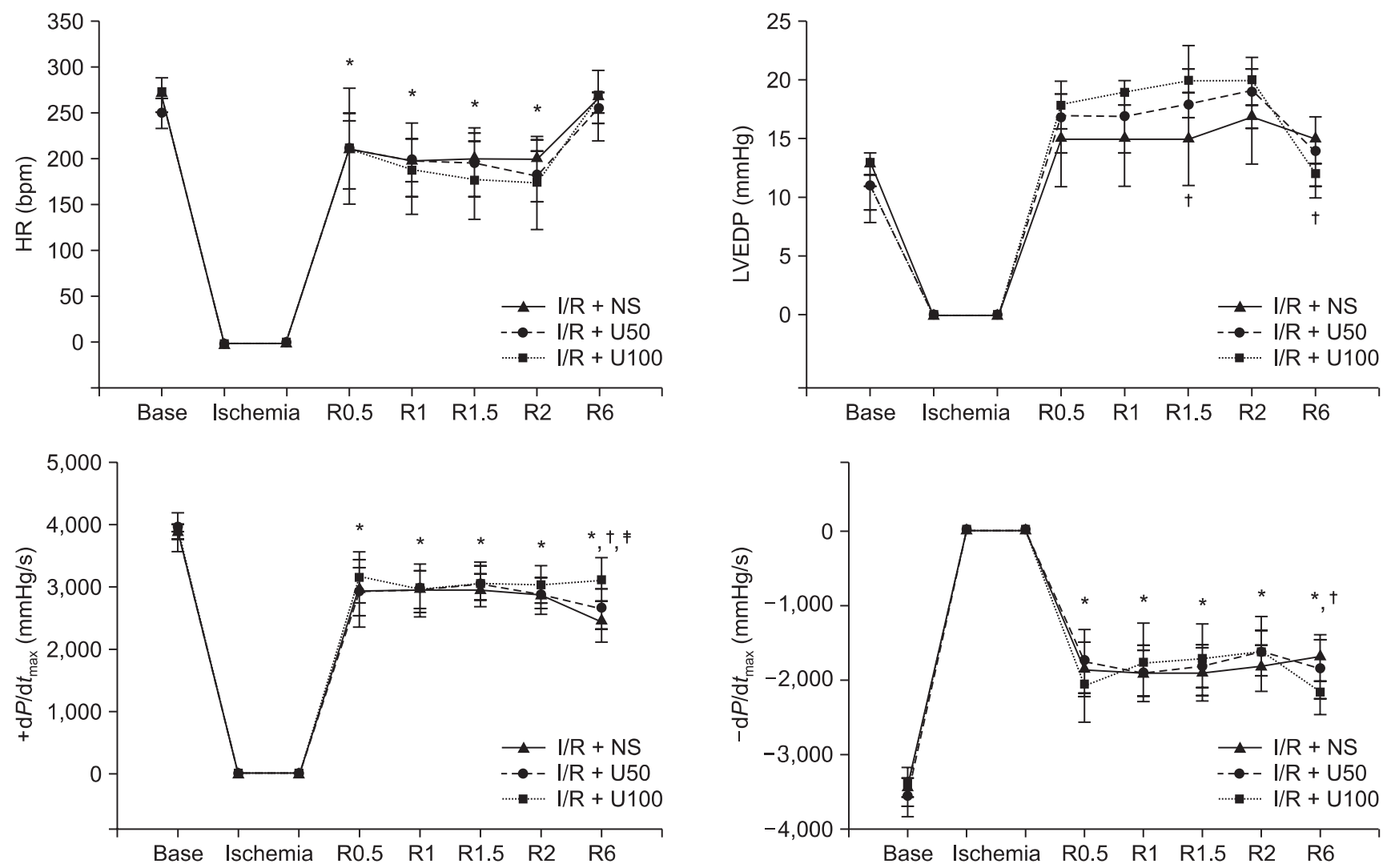

Fig. 1. Comparison of hemodynamic function in the I/R + NS, I/R + U50, and I/R + U100 groups. Values are means \pm SD ( $n=10 /$ group). The $\mathrm{I} / \mathrm{R}+\mathrm{NS}$ group - rats in which $1 \mathrm{ml}$ of NS was administered intraperitoneally $30 \mathrm{~min}$ before the ischemic insult; I/R + U50 group - rats in which $50,000 \mathrm{U} / \mathrm{kg}$ of ulinastatin diluted with NS was administrated intraperitoneally 30 min before the ischemic insult; I/R + U100 - rats in which $100,000 \mathrm{U} / \mathrm{kg}$ of ulinastatin diluted with NS was administered intraperitoneally $30 \mathrm{~min}$ before the ischemic insult. HR, heart rate; LVEDP, left ventricular end diastolic pressure; I/R, ischemia/reperfusion; NS, normal saline. R0.5, 30 min after reperfusion; R1, 1 h after reperfusion; R1.5, $1 \mathrm{~h} 30$ min after reperfusion; R2, $2 \mathrm{~h}$ after reperfusion; R6, $6 \mathrm{~h}$ after reperfusion. ${ }^{*} \mathrm{P}<0.05$ compared to basal hemodynamic function in the I/R + NS group. ${ }^{\dagger} \mathrm{P}<0.05$ compared to the I/R + NS group. ${ }^{\dagger} \mathrm{P}<0.05$ compared to the $\mathrm{I} / \mathrm{R}+\mathrm{U} 50$ group.

groups $(33 \pm 7 \%, \mathrm{P}<0.001)$ (Fig. 2$)$.

\section{Serum CK and cTnl levels}

I/R induced a significant increase in the serum level of CK after $6 \mathrm{~h}$ reperfusion. The elevation of serum CK concentration in the $\mathrm{I} / \mathrm{R}+\mathrm{NS}$ group $(200 \pm 40 \mathrm{U} / \mathrm{L})$ was significantlt inhibited in the $\mathrm{I} / \mathrm{R}+\mathrm{U} 50(149 \pm 45 \mathrm{U} / \mathrm{L}, \mathrm{P}=0.012)$ and $\mathrm{I} / \mathrm{R}+\mathrm{U} 100(114$ $\pm 35 \mathrm{U} / \mathrm{L}, \mathrm{P}<0.001$ ) groups after regional I/R injury (Fig. 3). I/ $\mathrm{R}$ induced a significant increase in serum levels of cTnI after 6 $\mathrm{h}$ reperfusion. The elevation of serum cTnI concentration in the $\mathrm{I} / \mathrm{R}+\mathrm{NS}$ group $(20 \pm 4 \mathrm{ng} / \mathrm{ml})$ were significantly higher than in the I/R + U50 (16 $\pm 3 \mathrm{ng} / \mathrm{ml}, \mathrm{P}=0.043)$ and I/R + U100 (14 \pm 4 $\mathrm{ng} / \mathrm{ml}, \mathrm{P}=0.012$ ) groups after regional I/R injury (Fig. 4).

\section{Plasma MPO and TNF- $\alpha$ activity}

$\mathrm{I} / \mathrm{R}$ induced a significant increase in the plasma level of MPO after $6 \mathrm{~h}$ reperfusion. The elevation of plasma MPO concentration in the I/R + NS group $(3,760 \pm 60 \mathrm{ng} / \mathrm{ml})$ were significantly higher than the I/R + U50 $(3,572 \pm 84 \mathrm{ng} / \mathrm{ml}, \mathrm{P}=$ $0.041)$ and $\mathrm{I} / \mathrm{R}+\mathrm{U} 100(2,848 \pm 120 \mathrm{ng} / \mathrm{ml}, \mathrm{P}<0.001)$ groups after regional I/R injury (Fig. 5). I/R induced a significant increase in the plasma level of TNF- $\alpha$ after $6 \mathrm{~h}$ reperfusion. The elevation of plasma TNF- $\alpha$ in the I/R + NS group (18 $\pm 1.2 \mathrm{ng} /$ $\mathrm{ml})$ was significantly greater than the $\mathrm{I} / \mathrm{R}+\mathrm{U} 50(16 \pm 0.9 \mathrm{ng} /$ $\mathrm{ml}, \mathrm{P}=0.042)$ and $\mathrm{I} / \mathrm{R}+\mathrm{U} 100(13 \pm 0.6 \mathrm{ng} / \mathrm{ml}, \mathrm{P}<0.001)$ groups after regional I/R injury (Fig. 6).

\section{Discussion}

Myocardial I/R injury is a major event in many clinical situations including the perioperative period, coronary thrombolysis, percutaneous coronary intervention, coronary artery bypass graft, and cardiac transplantation [16-18]. The non-cardiac surgical patient at risk of myocardial I/R injury is growing because of perioperative morbidity and mortality $[16,17]$. In this situation, applying the appropriate agents to reduce myocardial 


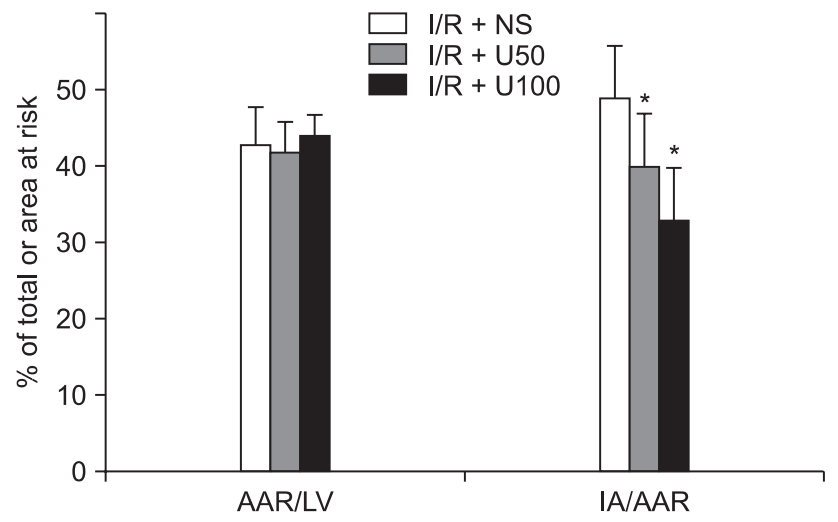

Fig. 2. Comparison of myocardial infarct size in the $I / R+N S, I / R+$ U50, and I/R + U100 groups. Values are means \pm SD ( $n=10 /$ group). The I/R + NS group - rats in which $1 \mathrm{ml}$ NS was administered intraperitoneally $30 \mathrm{~min}$ before the ischemic insult; I/R + U50 group - rats in which 50,000 U/ kg ulinastatin diluted with NS was administrated intraperitoneally $30 \mathrm{~min}$ before the ischemic insult; $\mathrm{I} / \mathrm{R}+\mathrm{U} 100$ - rats in which 100,000 U/kg ulinastatin diluted with NS was administered intraperitoneally $30 \mathrm{~min}$ before the ischemic insult. AAR, area at risk; LV, left ventricle; IA, infracted area; I/R, ischemia/reperfusion; NS, normal saline. ${ }^{*} \mathrm{P}<0.05$ compared to the $\mathrm{I} / \mathrm{R}+\mathrm{NS}$ group.

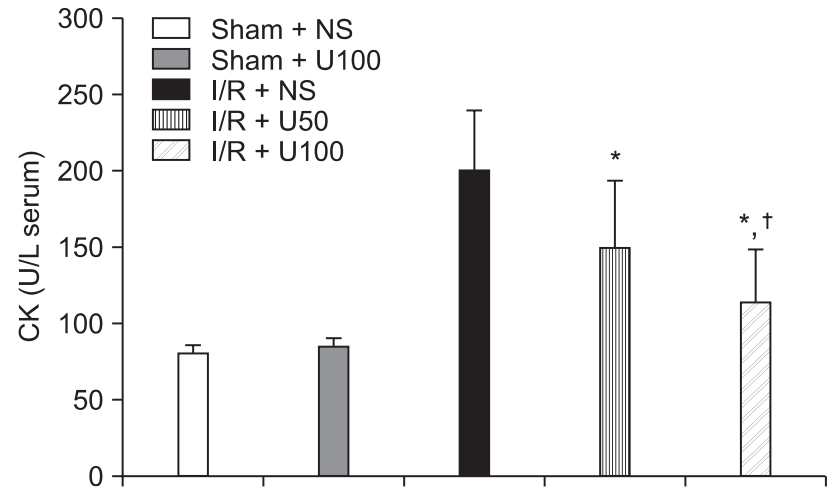

Fig. 3. Serum levels of creatine kinase at $6 \mathrm{~h}$ post-reperfusion. Values are means $\pm S D(n=6$ /group). The $I / R+N S$ group - rats in which $1 \mathrm{ml}$ NS was administered intraperitoneally $30 \mathrm{~min}$ before the ischemic insult; I/R + U50 group - rats in which 50,000 U/ kg ulinastatin diluted with NS was administrated intraperitoneally $30 \mathrm{~min}$ before the ischemic insult; I/R + U100 - rats in which 100,000 U/kg ulinastatin diluted with NS was administered intraperitoneally $30 \mathrm{~min}$ before the ischemic insult. CK, creatine kinase; $I / R$, ischemia/reperfusion; NS, normal saline. ${ }^{*} \mathrm{P}<0.05$ compared to the I/R + NS group. ${ }^{\dagger} \mathrm{P}<0.05$ compared to the I/R + U50 group.

I/R injury is critical. Thus, considerable research has been made to determine means to protect against myocardial injury. This study describes the benefit of ulinastatin as a myocardial protective agent.

Ulinastatin improved myocardial contractility, reduced myocardial infarct size, and decreased serum levels of CK and cTnI after regional I/R injury in an in vivo rat heart model. This protection appears to be, at least in part, due to inhibition of

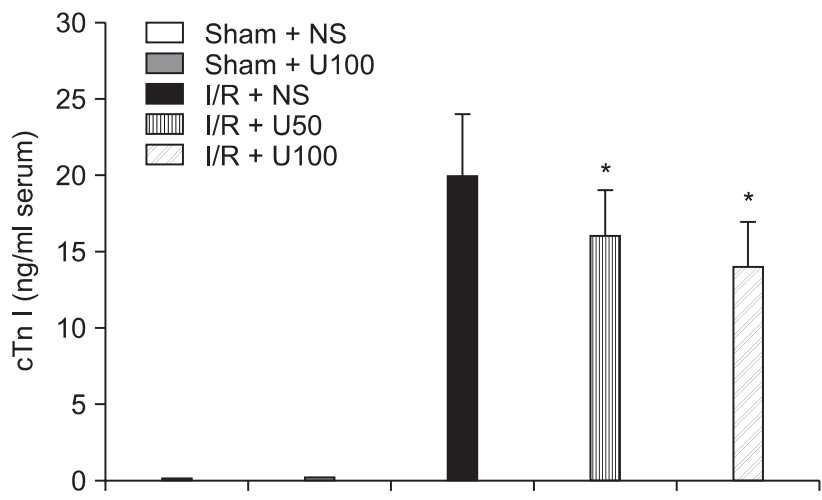

Fig. 4. Serum levels of cTnI at $6 \mathrm{~h}$ post-reperfusion. Values are means $\pm \mathrm{SD}$ ( $\mathrm{n}=6$ /group). Sham + NS group - rats in which $1 \mathrm{ml}$ of NS was administered intraperitoneally $30 \mathrm{~min}$ before operation without tightening of the left coronary artery; sham + U100 - rats in which $100,000 \mathrm{U} / \mathrm{kg}$ of ulinastatin diluted with NS was administered intraperitoneally $30 \mathrm{~min}$ before operation without tightening of the left coronary artery; I/R + NS - rats in which $1 \mathrm{ml}$ of NS was administered intraperitoneally $30 \mathrm{~min}$ before the ischemic insult; I/R + U50 group - rats in which $50,000 \mathrm{U} / \mathrm{kg}$ of ulinastatin diluted with NS was administrated intraperitoneally $30 \mathrm{~min}$ before the ischemic insult; $\mathrm{I} / \mathrm{R}+\mathrm{U} 100$ - rats in which 100,000 U/kg of ulinastatin diluted with NS was administered intraperitoneally $30 \mathrm{~min}$ before the ischemic insult. cTnI, cardiac troponin-I; I/R, ischemia/reperfusion; NS, normal saline. ${ }^{*} \mathrm{P}<0.05$ compared to the $\mathrm{I} / \mathrm{R}+\mathrm{NS}$ group.

TNF- $\alpha$ and MPO activities, which are indexes of tissue neutrophil infiltration and activation.

Myocardial infarction causes neutrophil infiltration into the infarct zone and increases the production of pro-inflammatory cytokines such as TNF- $\alpha$ [19-21]. Accumulation of neutrophils is evidence of acute inflammation and the infiltration of neutrophil can initiates myocardial damage $[19,20]$. The MPO activity assay is widely used to a biomarker for neutrophil infiltration and inflammatory reactivity, as MPO is an enzyme that is released mainly from neutrophils. Considering the attenuated MPO activity in the present study, ulinastatin may exert a myocardial protective effect by inhibiting the inflammatory process.

A previous study showed that ulinastatin has anti-inflammatory and organ protective effects in various I/R injury models [4,7-13]. Especially, ulinastatin has a myocardial protective effect by various mechanisms in an in vivo rat heart model. In reperfusion after hemorrhagic shock, ulinastatin contributed to the recovery of cardiac function after reperfusion by reducing the severity of mitochondrial dysfunction and maintaining energy production [6]. Ulinastatin also improved functional recovery of the heart after long cardioplegic arrest in an in vitro rabbit heart model [7]. In this study, ulinastatin had a myocardial protective effect via an anti-inflammatory response. Our study also showed that ulinastatin had a myocardial protective effect in the regional I/R injury in an in vivo rat heart 


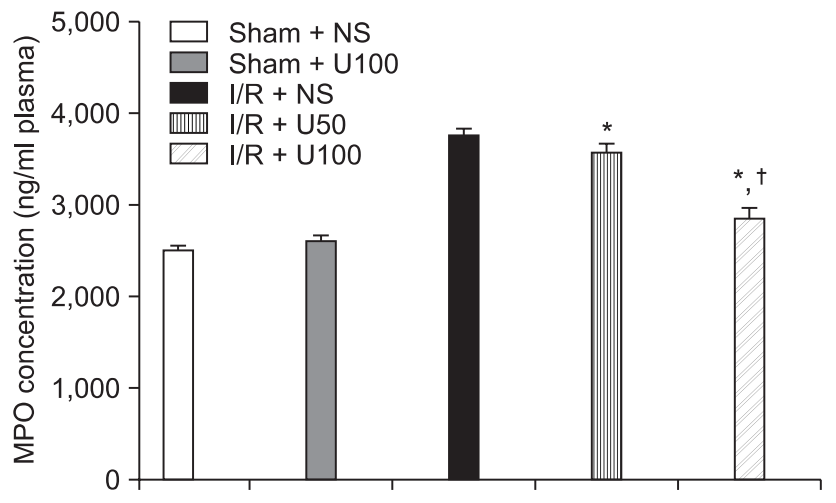

Fig. 5. Plasma levels of MPO at $6 \mathrm{~h}$ post-reperfusion. Values are means \pm SD ( $n=6$ /group). Sham + NS group - rats in which $1 \mathrm{ml}$ of NS was administered intraperitoneally $30 \mathrm{~min}$ before operation without tightening of the left coronary artery; sham + U100 - rats in which $100,000 \mathrm{U} / \mathrm{kg}$ of ulinastatin diluted with NS was administered intraperitoneally $30 \mathrm{~min}$ before operation without tightening of the left coronary artery; I/R + NS - rats in which $1 \mathrm{ml}$ of NS was administered intraperitoneally $30 \mathrm{~min}$ before the ischemic insult; I/ $\mathrm{R}+\mathrm{U} 50$ group - rats in which 50,000 U/ $\mathrm{kg}$ of ulinastatin diluted with NS was administrated intraperitoneally $30 \mathrm{~min}$ before the ischemic insult; I/R + U100 - rats in which 100,000 U/ kg of ulinastatin diluted with NS was administered intraperitoneally $30 \mathrm{~min}$ before the ischemic insult. MPO, myeloperoxidase; $\mathrm{I} / \mathrm{R}$, ischemia/reperfusion; NS, normal saline. ${ }^{*} \mathrm{P}<0.05$ compared to the I/R + NS group. ${ }^{\dagger} \mathrm{P}<$ 0.001 compared to the $\mathrm{I} / \mathrm{R}+\mathrm{U} 50$ group.

model.

Measurements of endogenous mediator, TNF- $\alpha$, allowed to elucidate the inflammatory response pathway. TNF- $\alpha$ is a potent pro-inflammatory cytokine that acts on vascular endothelial cells to induce expression of leukocyte adhesion molecules $[20,22]$. TNF- $\alpha$ is upregulated in the myocardium in response to a variety of cardiac injuries, including transient myocardial ischemia and reperfusion [22]. This suggests the possibility that TNF- $\alpha$ plays an important role in vivo in the pathogenesis of post-ischemic myocardial inflammation and I/R injury. In the present study, the significant reduction of TNF- $\alpha$ after ulinastatin suggests ulinastatin's possible myocardial protective effect via the inhibition of pro-inflammatory cytokine release.

Taken together, our results suggest that an anti-inflammatory effect is an important mechanism for myocardial protection following regional I/R injury in an in vivo rat heart ischemia model. The dose of ulinastatin was higher compared with clinically relevant doses. In this experiment, we used 50,000 and 100,000 $\mathrm{U} / \mathrm{kg}$ of ulinastatinn because these dosages had an organ protective effect in previous studies [6,9,10].

Our study has some limitations. First, necrotic cell death reaches a peak at $24 \mathrm{~h}$ after I/R injury, whereas apoptotic cell death develops progressively until $72 \mathrm{~h}$ [23]. This study observed the myocardial protective effects after $6 \mathrm{~h}$ of reperfusion. Therefore, long-term observations will be needed to determine whether

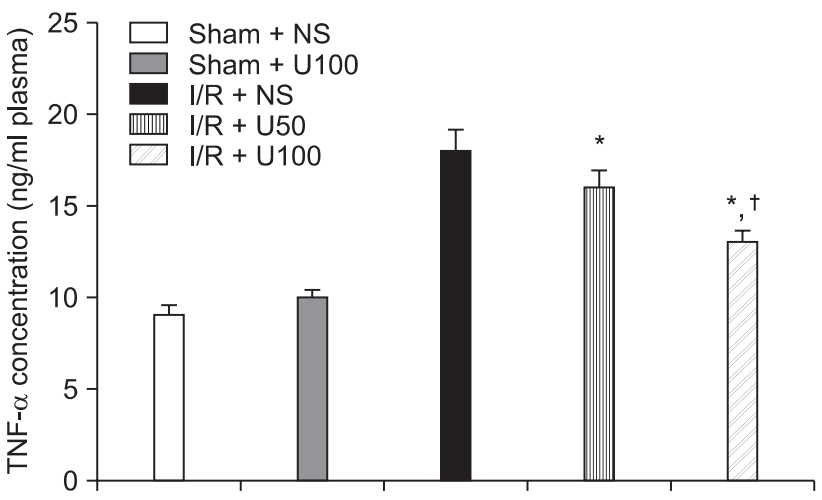

Fig. 6. Plasma levels of TNF- $\alpha$ at $6 \mathrm{~h}$ post-reperfusion. Values are means $\pm S D(n=6$ /group). Sham + NS group - rats in which $1 \mathrm{ml}$ of NS was administered intraperitoneally $30 \mathrm{~min}$ before operation without tightening of the left coronary artery, sham + U100: rats in which $100,000 \mathrm{U} / \mathrm{kg}$ of ulinastatin diluted by NS was administered intraperitoneally $30 \mathrm{~min}$ before operation without tightening of the left coronary artery, I/R + NS: rats in which $1 \mathrm{ml}$ of NS was administered intraperitoneally $30 \mathrm{~min}$ before the ischemic insult, I/ $\mathrm{R}+\mathrm{U} 50$ group: rats in which $50,000 \mathrm{U} / \mathrm{kg}$ of ulinastatin diluted by NS was administrated intraperitoneally $30 \mathrm{~min}$ before the ischemic insult, I/R + U100: rats in which 100,000 U/kg of ulinastatin diluted by NS was administered intraperitoneally $30 \mathrm{~min}$ before the ischemic insult. TNF- $\alpha$ : tumor necrosis factor- $\alpha, \mathrm{I} / \mathrm{R}$ : ischemia/reperfusion, NS: normal saline. ${ }^{*} \mathrm{P}<0.05$ compared to the $\mathrm{I} / \mathrm{R}+\mathrm{NS}$ group. ${ }^{\dagger} \mathrm{P}<$ 0.001 compared to the $\mathrm{I} / \mathrm{R}+\mathrm{U} 50$ group.

the effect is temporary or sustained. Second, ulinastatin was administered intraperitoneally and lacked a documented serum drug level. Therefore, this study cannot confirm that a clinically relevant ulinastatin concentration was associated with a myocardial protective effect.

In conclusion, ulinastatin reduced cardiac dysfunction, infarct size, MPO activity, and TNF- $\alpha$ release after regional I/R injury in an in vivo rat heart model. Its protective effect may be attributable to its anti-inflammatory.

\section{Acknowledgements}

This work was supported by the Korea Research Foundation Grant funded by the Korean Government (MOEHRD) (KRF2008-331-E00270).

\section{References}

1. Davis AE 3rd, Mejia P, Lu F. Biological activities of C1 inhibitor. Mol Immunol 2008; 45: 4057-63.

2. Owen CA, Campbell EJ. The cell biology of leukocyte-mediated proteolysis. J Leukoc Biol 1999; 65: 137-50.

3. Shibata T, Yamamoto F, Suehiro S, Kinoshita H. Effects of protease inhibitors on postischemic recovery of the heart. Cardiovasc Drugs Ther 1997; 11: 547-56.

4. Harada N, Okajima K, Kushimoto S. Gabexate mesilate, a synthetic 
protease inhibitor, reduces ischemia/reperfusion injury of rat liver by inhibiting leukocyte activation. Crit Care Med 1999; 27: 1958-64.

5. Pugia MJ, Lott JA. Pathophysiology and diagnostic value of urinary trypsin inhibitors. Clin Chem Lab Med 2005; 43: 1-16.

6. Masuda T, Sato K, Noda C, Ikeda KM, Matsunaga A, Ogura MN, et al. Protective effect of urinary trypsin inhibitor on myocardial mitochondria during hemorrhagic shock and reperfusion. Crit Care Med 2003; 31: 1987-92.

7. Cao ZL, Okazaki Y, Naito K, Ueno T, Natsuaki M, Itoh T. Ulinastatin attenuates reperfusion injury in the isolated blood-perfused rabbit heart. Ann Thorac Surg 2000; 69: 1121-6.

8. Yano T, Anraku S, Nakayama R, Ushijima K. Neuroprotective effect of urinary trysin inhibitor against focal cerebral ischemia-reperfusion injury in rats. Anesthesiology 2003; 98: 465-73.

9. Koga Y, Fujita M, Tsuruta R, Koda Y, Nakahara T, Yagi T, et al. Urinary trypsin inhibitor suppresses excessive superoxide anion radical generation in blood, oxidative stress, early inflammation, and endothelial injury in forebrain ischemia/reperfusion rats. Neurol Res 2010; 32: 925-32.

10. Wu YJ, Ling Q, Zhou XH, Wang Y, Xie HY, Yu JR, et al. Urinary trypsin inhibitor attenuates hepatic ischemia-reperfusion injury by reducing nuclear factor-kappa B activation. Hepatobiliary Pancreat Dis Int 2009; 8: 53-8.

11. Aihara T, Shiraishi M, Hiroyasu S, Hatsuse K, Mochizuki H, Seki S, et al. Ulinastatin, a protease inhibitor, attenuates hepatic ischemia/ reperfusion injury by downregulating TNF-alpha in the liver. Transplant Proc 1998; 30: 3732-4.

12. Chen CC, Liu ZM, Wang HH, He W, Wang Y, Wu WD. Effects of ulinastatin on renal ischemia-reperfusion injury in rats. Acta Phamacol Sin 2004; 25: 1334-40.

13. Ren B, Wu H, Zhu J, Li D, Shen Y, Ying R, et al. Ulinastatin attenuates lung ischemia-reperfusion injury in rats by inhibiting tumor necrosis factor alpha. Transplant Proc 2006; 38: 2777-9.

14. Shin IW, Jang IS, Lee SH, Baik JS, Park KE, Sohn JT, et al. Propofol has delayed myocardial protective effects after a regional ischemia/ reperfusion injury in an in vivo rat heart model. Korean J Anesthesiol 2010; 58: 378-82.

15. Jang IS, Park MY, Shin IW, Sohn JT, Lee HK, Chung YK. Ethyl pyruvate has anti-inflammatory and delayed myocardial protective effects after regional ischemia/reperfusion injury. Yonsei Med J 2010; 51 : 838-44.

16. Mangano DT. Perioperative cardiac morbidity. Anesthesiology 1990; 72: 153-84.

17. Badner NH, Knill RL, Brown JE, Novick TV, Gelb AW. Myocardial infarction after noncardiac surgery. Anesthesiology 1998; 88: 572-8.

18. Mark DB, Nelson CL, Califf RM, Harrell FE Jr, Lee KL, Jones RH, et al. Continuing evolution of therapy for coronary artery disease. Initial results from the era of coronary angioplasty. Circulation 1994; 89: 2015-25.

19. Hansen PR. Role of neutrophils in myocardial ischemia and reperfusion. Circulation 1995; 91: 1872-85.

20. Frangogiannis NG, Smith CW, Entman ML. The inflammatory response in myocardial infarction. Cardiovasc Res 2002; 53: 31-47.

21. Goldmann BU, Rudolph V, Rudolph TK, Holle AK, Hillebrandt M, Meinertz T, et al. Neutrophil activation precedes myocardial injury in patients with acute myocardial infarction. Free Radic Biol Med 2009; 47: 79-83.

22. Herskowitz A, Choi S, Ansari AA, Wesselingh S. Cytokine mRNA expression in postischemic/reperfused myocardium. Am J Pathol 1995; 146: 419-28.

23. Zhao ZQ, Velez DA, Wang NP, Hewan-Lowe KO, Nakamura M, Guyton RA, et al. Progressively developed myocardial apoptotic cell death during late phase of reperfusion. Apoptosis 2001; 6: 279-90. 